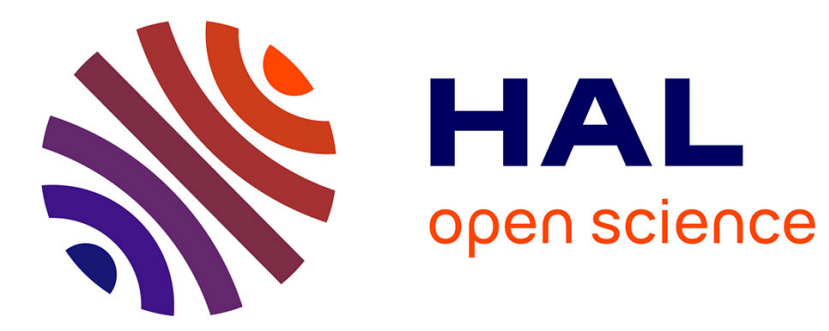

\title{
Numerical simulation of unsteady MHD flows and applications
}

\author{
Remi Abgrall, Pierre Ramet, Robin Huart
}

\section{To cite this version:}

Remi Abgrall, Pierre Ramet, Robin Huart. Numerical simulation of unsteady MHD flows and applications. Magnetohydrodynamics c/c of Magnitnaia Gidrodinamika, 2009, 45 (2), pp.225-232. hal00547413

\section{HAL Id: hal-00547413 \\ https://hal.science/hal-00547413}

Submitted on 16 Dec 2010

HAL is a multi-disciplinary open access archive for the deposit and dissemination of scientific research documents, whether they are published or not. The documents may come from teaching and research institutions in France or abroad, or from public or private research centers.
L'archive ouverte pluridisciplinaire HAL, est destinée au dépôt et à la diffusion de documents scientifiques de niveau recherche, publiés ou non, émanant des établissements d'enseignement et de recherche français ou étrangers, des laboratoires publics ou privés. 


\title{
Numerical simulation of unsteady MHD flows and applications
}

\author{
R. Abgrall ${ }^{1,2}$, R. Huart ${ }^{1}$, P. Ramet Ra $^{1,3}$ \\ 1 INRIA Bordeaux Sud-Ouest \\ ${ }^{2}$ Mathématiques Appliquées de Bordeaux, Université Bordeaux 1 \\ ${ }^{3}$ LABRI, Université Bordeaux 1
}

We present a robust numerical method for solving the compressible Ideal MagnetoHydrodynamic equations. It is based on the Residual Distribution (RD) algorithms already successfully tested in many problems [1]. We adapted the scheme to the multidimensional unsteady MHD model. The constraint $\boldsymbol{\nabla} \cdot \boldsymbol{B}=0$ is enforced by the use a Generalized Lagrange Multiplier (GLM) technique [2]. First, we present this complete system and the keys to get its eigensystem, as we may need it in the algorithm. Next, we introduce the numerical scheme built in order to get a compressible, unsteady and implicit solver which has good shock-capturing properties and is second-order accurate at the converged state. To show the efficiency of our method, we will then comment some $2 \mathrm{D}$ results. We will end by pointing out some issues and the extensions we plan for this solver.

1. Introduction The interest in the simulation of plasma models, and so in the resolution of the Magneto-Hydrodynamic equations, has been much increasing in the last decade due to the rise of new techniques and fields of research like thermonuclear fusion or in astrophysics. Our present work here is situated in the ITER context, as we aim to be soon able to simulate plasma instabilities, but it is expected to be of the same interest for all applications of the MHD model. We won't talk about the recent addition of the resistive MHD in the solver, because it has not been tested yet. Up to now, lots of numerical methods have been tested for solving the compressible MHD equations. However, most of them rely on approximate Riemann solvers used in standard Godunov schemes. Here we want to improve the ability of Residual Distribution schemes to solve this hyperbolic system, as started earlier in [3]. In this last reference, the authors mainly tested the LDA and $\mathrm{N}$ schemes which are two genuinely multidimensional upwind RD schemes. The most promising results were obtained by making a modified $\mathrm{N}$ scheme, another upwind RD scheme. Here we focus on the limited and stabilized Rusanov scheme [4], which captures shocks very well, as well as other discontinuities as slip lines or very strong gradient zones, and is much easier to implement than the previous N, LDA and blended RD schemes. We add a correction technique to ensure the solenoidal character of the magnetic field. Multidimensional upwinding techniques are made possible by the derivation of the full eigensystem of the corrected symmetrizable system. We indicate here the way we made that, through the symmetrization of the equations. Following the idea of [3], we use the system with Powell's source terms [5] while doing that, i.e. each time we use the eigensystem, whereas we solve the equations without them.

Let us now review first the whole corrected model and its symmetrization, and after the numerical method we employed. Then we will comment the $2 \mathrm{D}$ results we have got until now. While concluding, we'll also point out the improvements still to be done and what we plan to add to our model. 
2. Description of the method Let us recall the physical context of our work and then we present the numerical method used to deal with it.

2.1. The Ideal MHD equations Naturally, we started with studying and discretizing these advection PDEs. It describes the behavior of a totally ionized gas, called a plasma. The approximation of a perfect fluid, underlying the Ideal MHD model, here partly means that the plasma has no viscosity nor resistivity: it's a perfectly conducting gas. As in traditional fluid mechanics, this comes from neglecting some collision effects. We also add an equation of state of perfect gases $p=(\gamma-1) \rho e$, which is here a sufficiently good approximation, with $p$ the hydrodynamic pressure, $\rho e=E-\frac{1}{2} \rho \boldsymbol{u}^{2}-\frac{B^{2}}{2 \mu_{0}}$ and $E$ the total energy.

We may note from now on that we won't make any use of the total pressure, usually called $P=p+\frac{B^{2}}{2 \mu_{0}}$, in order to avoid confusions. In the rest of this paper, we will drop the magnetic permeability $\mu_{0}$ by assuming that the magnetic field $\boldsymbol{B}$ has been normalized in this way : $\boldsymbol{B}:=\frac{\boldsymbol{B}}{\sqrt{\mu_{0}}}$.

We start with the conservative form of the Ideal MHD equations, adding another equation which is not explicitly included in this system : the Maxwellflux equation $\operatorname{div} \boldsymbol{B}=0$. Many ideas were found to ensure this constraint during the simulations. Mainly two techniques among them revealed to give good results

1. Building divergence-free elements by a collocation method, consisting in storing the magnetic field values on the faces/edges of each element (for a vertexcentered approach),

2. Introducing some correction terms by modifying the system of equations and adding a scalar variable representing the divergence of the magnetic field.

The first approach has shown to ensure a very accurate zero divergence. However, this technique is not suitable in a Residual Distribution framework. The use of a correcting variable coupled to the equations was then the best choice. Following the works of Dedner, Munz et al. [2], we modified the system

$$
\begin{gathered}
\frac{\partial \rho}{\partial t}+\nabla \cdot(\rho \boldsymbol{u})=0 \\
\frac{\partial(\rho \boldsymbol{u})}{\partial t}+\boldsymbol{\nabla} \cdot\left(\rho \boldsymbol{u} \boldsymbol{u}^{t}+\left(p+\frac{\boldsymbol{B}^{2}}{2}\right) I d-\boldsymbol{B B}^{t}\right)=\mathbf{0} \\
\frac{\partial E}{\partial t}+\boldsymbol{\nabla} \cdot\left(\left(E+p+\frac{\boldsymbol{B}^{2}}{2}\right) \boldsymbol{u}-(\boldsymbol{u} \cdot \boldsymbol{B}) \boldsymbol{B}\right)=0 \\
\frac{\partial \boldsymbol{B}}{\partial t}+\boldsymbol{\nabla} \cdot\left(\boldsymbol{u} \boldsymbol{B}^{t}-\boldsymbol{B} \boldsymbol{u}^{t}+\psi I d\right)=\mathbf{0} \\
\frac{\partial \psi}{\partial t}+c_{h}{ }^{2} \boldsymbol{\nabla} \cdot \boldsymbol{B}=-\frac{c_{h}{ }^{2}}{c_{p}{ }^{2}} \psi
\end{gathered}
$$

Both classical and new systems can be reformulated in a quasi-linear form involving diagonalizable Jacobean matrices. This means they are hyperbolic systems of PDEs, as we only need to work with real quantities.

$$
\frac{\partial U}{\partial t}+\boldsymbol{K} \cdot \nabla U=0
$$

where $\boldsymbol{K}=(A B C)^{t}$ is a vector of Jacobian matrices, $U$ being the vector of the conservative variables.

Considering an arbitrary direction $\boldsymbol{n}$, we are interested in studying $K_{n}$, the projection of the Jacobian matrices. The eigenvalues of $K_{n}$ for the corrected system consist of the classical transport, slow and fast magneto-sonic and Alfvén 
waves, and of new divergence waves. Respectively $\lambda_{1}=u_{n}, \lambda_{ \pm s}=u_{n} \pm c_{s}$, $\lambda_{ \pm f}=u_{n} \pm c_{f}, \lambda_{ \pm a}=u_{n} \pm c_{a}, \lambda_{ \pm h}= \pm c_{h}$ where $u_{n}$ is the dot product of the velocity and the vector $\vec{n}$.

$$
\begin{aligned}
& \text { Noting } a=\sqrt{\frac{\gamma p}{\rho}}, b=\sqrt{\frac{B^{2}}{\rho}} \text { and } b_{n}=\frac{B \cdot n}{\sqrt{\rho}} \text {, the wave speeds are given by } c_{s}= \\
& \sqrt{\frac{1}{2}\left(a^{2}+b^{2}-\sqrt{\left(a^{2}+b^{2}\right)^{2}-4 a^{2} b_{n}{ }^{2}}\right)}, c_{f}=\sqrt{\frac{1}{2}\left(a^{2}+b^{2}+\sqrt{\left(a^{2}+b^{2}\right)^{2}-4 a^{2} b_{n}^{2}}\right)} \\
& \text { and } c_{a}=b_{n} .
\end{aligned}
$$

Remark 2.1 Alfvén waves only appear in the three-dimensional case.

As we may need some characteristic decompositions in the numerical methods, we are also looking for the full eigensystem of $K_{n}$, that is to say the left and right eigenvectors. Unfortunately, the direct formal calculation of these vectors is really complex. It was early found [6] that the Ideal MHD system could be symmetrized by adding some source terms proportional to $\boldsymbol{\nabla} \cdot \boldsymbol{B}$, hence not modifying the physical solutions of the equations.

For the corrected system, adding the same source terms doesn't make it symmetrizable. In fact, if we look at the last two eigenvalues, we note that the system does not even achieve Galilean invariance. Then, in [2], the authors proposed to add some symmetrizing terms so that this last property be recovered, depending on the gradient of $\psi$.

Moreover, as we make unsteady computations, the time $t$ truly denotes the physical time and we aim to get a divergence-free solution at each physical time step. Then we drop the time derivative from the last equation (1b), which actually corresponds to the converged form of the equation : $\boldsymbol{\nabla} \cdot \boldsymbol{B}=-\frac{\psi}{c_{p}{ }^{2}}$. This will provide a definition of our numerical divergence.

The Jacobian $K_{n}^{*}$ of the symmetrizable system is

$$
K_{n}^{*}=\left(\begin{array}{ccccc}
0 & \boldsymbol{n}^{t} & 0 & \mathbf{0}^{t} & 0 \\
(\gamma-1) \frac{\boldsymbol{u}^{2}}{2} \boldsymbol{n}-u_{n} \boldsymbol{u} & (1-\gamma) \boldsymbol{n} \boldsymbol{u}^{t}+\boldsymbol{u} \boldsymbol{n}^{t}+u_{n} I d & (\gamma-1) \boldsymbol{n} & (2-\gamma) \boldsymbol{n} \boldsymbol{B}^{t}-B_{n} I d & \mathbf{0} \\
\left(K_{n}^{*}\right)_{5,1} & \frac{H}{\rho} \boldsymbol{n}^{t}+(1-\gamma) u_{n} \boldsymbol{u}^{t}-\frac{B_{n}}{\rho} \boldsymbol{B}^{t} & \gamma u_{n} & (2-\gamma) u_{n} \boldsymbol{B}^{t}-B_{n} \boldsymbol{u}^{t} & B_{n} \\
\frac{B_{n} \boldsymbol{u}-u_{n} \boldsymbol{B}}{\rho} & \frac{\boldsymbol{B} \boldsymbol{n}^{t}-B_{n} I d}{\rho} & \mathbf{0} & u_{n} I d & \boldsymbol{n} \\
0 & \mathbf{0}^{t} & 0 & c_{h}{ }^{2} \boldsymbol{n}^{t} & u_{n}
\end{array}\right)
$$

where $H=E+p+\frac{B^{2}}{2}$ is the enthalpy, $c=\sqrt{\frac{\gamma p}{\rho}}$ the classical sound speed for the Euler equations, and $\left(K_{n}^{*}\right)_{5,1}=u_{n}\left((\gamma-1) \frac{\boldsymbol{u}^{2}}{2}-\frac{H}{\rho}\right)+\frac{B_{n}}{\rho} \boldsymbol{u} \cdot \boldsymbol{B}$.

As we said the system was verifying Galilean invariance (we'll see it after the symmetrization), the last eigenvalue has become $\lambda_{ \pm h}=u_{n} \pm c_{h}$.

2.2. A note on the role of $\psi$. Like in [2], we prefer using the generalized Lagrange Multiplier (GLM) approach with a mixed hyperbolic-parabolic correction. It offers both transport and dissipative (smoothing) properties, which can be seen by rewriting the last equation in terms of $\psi$. First, one has to note that the divergence of equation (1a) gives

$$
\partial_{t}(\boldsymbol{\nabla} \cdot \boldsymbol{B})+\Delta \psi=0 .
$$

Then, taking the time derivative of equation (1b), we get the Telegraph equation

$$
\frac{\partial^{2} \psi}{\partial t^{2}}+\frac{c_{h}^{2}}{c_{p}^{2}} \frac{\partial \psi}{\partial t}-c_{h}^{2} \Delta \psi=0
$$


Consequently, the ratio $\frac{c_{h}}{c_{p}}$ gives a control on the balance between parabolic and hyperbolic behaviors.

2.3. Symmetrization Starting from the symmetrizable system, we followed Jameson's work [7] to get a set of symmetrizing variables $W$ satisfying

$$
d W=\left(\frac{d p}{c^{2}}, \frac{\rho}{c} d u, \frac{\rho}{c} d v, \frac{\rho}{c} d w, \frac{d p}{c^{2}}-d \rho, \frac{\sqrt{\rho}}{c} d B_{x}, \frac{\sqrt{\rho}}{c} d B_{y}, \frac{\sqrt{\rho}}{c} d B_{z}, \frac{\sqrt{\rho}}{c} \frac{d \psi}{c_{h}}\right)^{t} .
$$

Denoting $h=\frac{c^{2}}{\gamma-1}+\frac{\boldsymbol{u}^{2}}{2}$ and $\beta=\frac{\gamma-1}{c^{2}}$, the transformation matrices $\frac{\partial U}{\partial W}$ and $\frac{\partial W}{\partial U}$ are easily found. The symmetrized Jacobian is given by $K_{s}=\frac{\partial W}{\partial U} K_{n}^{*} \frac{\partial U}{\partial W}$

$$
K_{s}=\left(\begin{array}{ccccc}
u_{n} & c \boldsymbol{n}^{t} & 0 & \mathbf{0}^{t} & 0 \\
c \boldsymbol{n} & u_{n} I d & \mathbf{0} & \frac{n B^{t}-B_{n} I d}{\sqrt{\rho}} & 0 \\
0 & \mathbf{0}^{t} & u_{n} & \mathbf{0}^{t} & 0 \\
\mathbf{0} & \frac{\boldsymbol{B n ^ { t } - B _ { n } I d}}{\sqrt{\rho}} & \mathbf{0} & u_{n} I d & c_{h} \boldsymbol{n} \\
0 & \mathbf{0}^{t} & 0 & c_{h} \boldsymbol{n}^{t} & u_{n}
\end{array}\right)
$$

Here we see clearly that Galilean invariance is achieved, as $K_{s}=u_{n} I d+$ $\left.K_{s}\right|_{u_{n}=0}$. We are then able to calculate the full eigensystem of this matrix, and so of $K_{n}^{*}$.

2.4. The numerical scheme. Our framework is the RD approach, which principles are known as

1. Integrating the unsteady equations over the contour of an element $T$ to get the so-called "residual" $\Phi^{T}$,

2. Distributing it to each node of the element $\rightarrow \Phi_{i}^{T}$,

3. For each node of the mesh, adding all partial residuals and solving

$$
\sum_{T, i \in T} \Phi_{i}^{T}=0
$$

Details can be found in [4], we only recall here the more important points and how they adapt to the MHD equations.

While the first step basically relies on a quadrature formula conditioning the formal accuracy of the whole scheme, all RD schemes differ from each other by their second step. For our purpose, we only deal with the Rusanov scheme. The pure one consists in distributing the residuals in the following way

$$
\Phi_{i}^{T}=\frac{1}{N_{s}}\left(\Phi^{T}+\alpha \sum_{j \in T}\left(U_{i}-U_{j}\right)\right)
$$

where $N_{s}$ is the number of nodes in the element and $\alpha$ is the maximum of all the eigenvalues of $K_{n}$ (not $K_{n}^{*}$ !). Since $\alpha$ strongly conditions the behavior of the scheme, we have to take care about the value we impose for $c_{h}$ : it shouldn't not be greater than the fastest physical wave. Here, it means we have to choose $c_{h}<c_{f}$.

We prefer another definition for the unsteady Rusanov scheme

$$
\Phi_{i}^{T}=\frac{1}{N_{s}}\left(\left(\frac{\partial u^{h}}{\partial t}\right)_{i}+\int_{T} \operatorname{div} \boldsymbol{F}(U)+\alpha \sum_{j \in T}\left(U_{i}-U_{j}\right)\right)
$$


This distribution has very interesting properties [4]. Of course, it is necessarily conservative as $\sum_{i \in T} \Phi_{i}^{T}=\Phi^{T}$. But its main advantage remains in its strong positivity, ensured by the choice of $\alpha$, making it capturing shocks very well and in a monotonic manner.

For the time discretization, we choose the Gear implicit scheme so that we get, in the case of a constant time step $\Delta t$

$$
\begin{gathered}
\left(\frac{\partial u^{h}}{\partial t}\right)_{i}^{\text {Gear }}=\frac{3}{2} \frac{U_{i}^{n+1}-U_{i}^{n}}{\Delta t}-\frac{1}{2} \frac{U_{i}^{n}-U_{i}^{n-1}}{\Delta t} \\
\Phi_{i}^{T}=\frac{1}{N_{s}}\left(\left(\frac{\partial u^{h}}{\partial t}\right)_{i}^{G e a r}+\int_{T} \operatorname{div} \boldsymbol{F}(U)^{n+1}+\alpha \sum_{j \in T}\left(U_{i}^{n+1}-U_{j}^{n+1}\right)\right)
\end{gathered}
$$

However, it results in a first order scheme. In order to get, formally, the second order of accuracy, we have to turn it into a modified nonlinear scheme [4].

First, let us recall that all RD schemes can be rewritten like it : $\Phi_{i}^{T}=\beta_{i}^{T} \Phi^{T}$. The idea is to modify $\beta_{i}^{T}$ so that we ensure it is bounded, and then we know that $\Phi_{i}^{T}$ and $\Phi^{T}$ are of the same order of accuracy, e.g. second order if the quadrature formula is so. This nonlinear process is called the "Limitation". The bounding of these coefficients also improves the monotonicity of the solution. In fact, we can still improve this by projecting the residuals $\Phi_{i}^{T}$ in the basis of the eigenvectors of $K_{n}^{*}$, which provides better conditioned $\beta_{i}^{T}$. However, the limitation application does not preserve the solenoïdal character of the magnetic field, and that is why the correction technique is absolutely necessary even in simple test cases : with its use, the method effectively converges to a divergence-free behavior.

Second, we have to ensure that the convergence of the algorithm chosen for the third step will be good enough. In other words, we want a good conditioning for the system to solve. A way to do that, is to add a SUPG-like term $S_{i}^{T}$ providing an upwind bias to our discretization [4]. Indeed, the basic distribution of the Rusanov scheme doesn't respect upwinding and we have no control on it. That's why it may be even possible that a node doesn't receive information at all from the surrounding elements. Nevertheless, as the effect of this term is also to smooth out the regular solutions, we don't apply this near discontinuities. In fact we use a shock sensor $\theta^{T}$. This addition is called the "Stabilization". One possible expression is $S_{i}^{T}=-\frac{\theta^{T}}{d} K_{i} N \Phi^{T}$, where $d$ is the number of space dimensions and $N$ is the matrix of the Narrow scheme (see e.g. [1]), for which the knowledge of the eigensystem is required. The limited and stabilized Rusanov scheme, $\Phi_{i}^{T}=$ $\left(\beta_{i}^{R u s}\right)^{*} \Phi^{T}+S_{i}^{T}$, achieves second order of accuracy when converged.

We recall that we expect, like in [3], that the use of the symmetrizable (nonconservative) system in the limitation and the stabilization, instead of the eigensystem of the conservative equations (actually solved), should not affect the quality of the solution.

The third step, gathering all the signals and finding the zero for each nodeassociated problem, is performed by mean of an iterative Newton method, which solves a linear problem in each iteration.

\section{Results}

3.1. The Rotor problem This test, taken from [8], involves strong oblique Alfvén waves rising from a rotating high density region in the center of the domain. At the beginning, the ambient fluid is light and static. The whole domain is under 
the effect of an initially uniform magnetic field in the $x$-direction. The problem takes place in a two dimensional unit square containing $200 \times 200$ cells. The two parts of the plasma have the same pressure $p=1$, magnetic field $B_{x}=5$ and $B_{y}=0$. The central region, inside a radius $r=0.1$, has a 10 units density and spins at a constant rotating speed $\omega=2$. From $r=0.1$ to 0.115 , the density decreases linearly from 10 to 1 , so that we have no real discontinuity (to avoid a strong shock). The ratio of specific heats is $\gamma=1.4$, and we set $c_{h}=1$ and $c_{p}=10$. On Figure 1, we show the solution at time 0.18 , when the waves reach the boundary (arbitrarily considered as opened).

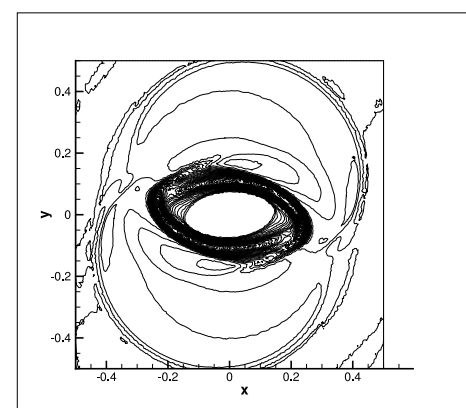

(a) Density.

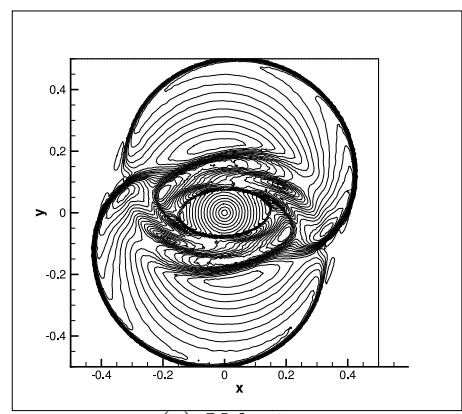

(c) Velocity.

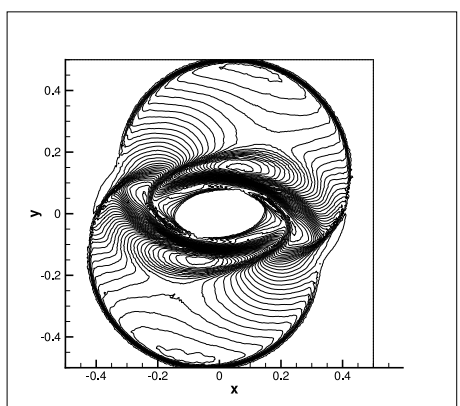

(b) Magnetic pressure.

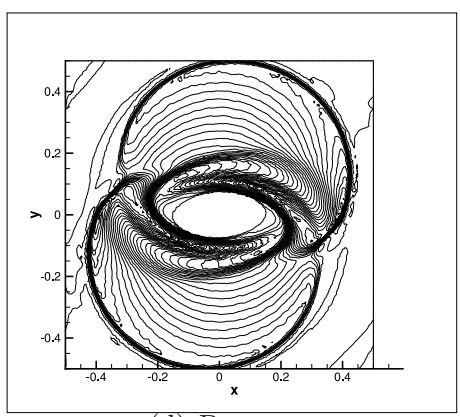

(d) Pressure.

Figure 1: A rotor involving torsional waves.

3.2. The Blast problem Also inspired from [8], this simulation introduces a very strong shock wave coming from a central high pressure zone. We use the same mesh as before. The plasma is entirely exposed to a strong magnetic field along the $x$-direction so that the particles, for most of them, are constrained to move parallel to it. Hence we expect to get two fronts moving along $x$ until they reach the opened boundaries. Initially, the uniform magnetic field is $B_{x}=100$ and $B_{y}=0$, the density is 1 and the velocity vector $\mathbf{0}$ everywhere. Inside the central zone, inside a radius $r=0.1$, we set the hydrodynamic pressure $p=1000$, which falls immediately to 0.1 outside this region. We keep the same $\gamma$ and we set $c_{h}=10$ and $c_{p}=100$. In Figure 2, we show the solution at time 0.1. This problem has been run without stabilization, causing the solution to wiggle a little.

4. Conclusion and prospectives We have got an efficient solver for $2 \mathrm{D}$ Ideal MHD problems. However, more tests will be necessary, and we will look for 


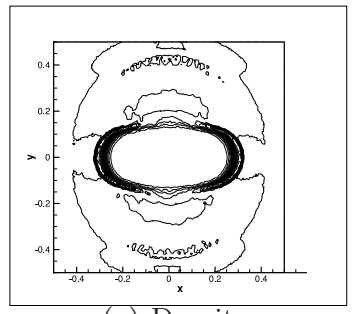

(a) Density.

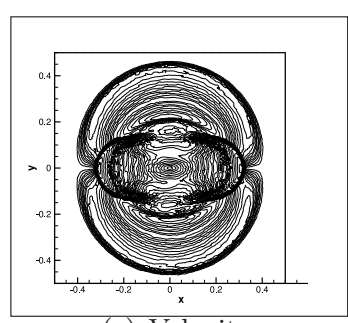

(c) Velocity

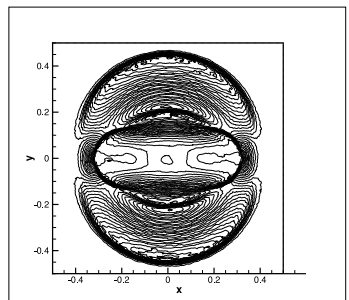

(b) Magnetic pressure.

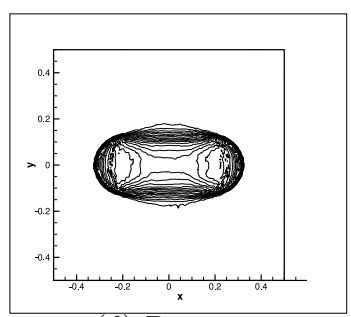

(d) Pressure.

Figure 2: A blast explosion with fast shock waves.

possible improvements of the stabilization procedure. The code is also expected to work in three dimensional cases. We started to implement the resistive MHD equations by using a Continuous Galerkin method, which has not been tested yet. We aim at simulating plasma instabilities for ITER configurations.

Acknowledgements We really want to thank M. Ricchiuto and A. Larat from INRIA for their support during all this work. This project has been financed by the ANR CIS ASTER (http://aster.gforge.inria.fr/index.html).

\section{References}

1. R. AbGrall. Toward the ultimate conservative scheme : following the quest. $J$. Comput. Phys., vol. 167 (2001), pp. 277-315.

2. A. Dedner, et AL. Hyperbolic divergence cleaning for the mhd equations. J. Comput. Phys., vol. 175 (2002), pp. 645-673.

3. A. Csik. Upwind residual distribution schemes for general hyperbolic conservation laws and application to ideal magnetohydrodynamics. Ph.D. thesis, Katholieke Universiteit Leuven, 2002.

4. R. AbGrall. Essentially non oscillatory residual distribution schemes for hyperbolic problems. J. Comput. Phys., vol. 214 (2006), no. 2, pp. 773-808.

5. K. G. Powell, Et AL. An upwind scheme for magnetohydrodynamics. J. Comput. Phys., vol. 154 (1999), pp. 284-309.

6. S. K. Godunov. Nonlinear hyperbolic problems : Proceedings of an advanced research workshop. In C. Carasso, P. A. Raviart, and D. Serre, editors, Lecture Notes in Mathematics (Springer-Verlag, Berlin, 1987), vol. 1270.

7. A. JAmeson. Eigenvalues, eigenvectors and symmetrization of the magnetohydrodynamic (mhd) equations. Tech. rep., AFSOR, 2006.

8. D. S. Balsara AND D. S. Spicer. A staggered mesh algorithm using high order godunov fluxes to ensure solenoidal magnetic fields in magnetohydrodynamics simulations. J. Comput. Phys., vol. 149 (1999), pp. 270-292. 